\title{
Prevenção de acidentes em crianças e adolescentes na estratégia saúde da família
}

\author{
Preventing accidents in children and teenagers in the family health strategy \\ Prevención de accidentes en niños y adolescentes en la estrategia de salud de la familia
}

\author{
Ana Cristina Pereira de Jesus Costal; Ester Cíntia Santiago Pereirall; Ana Larissa Gomes MachadoIII; \\ Álissan Karine Lima Martins ${ }^{I V}$; Patrícia Neyva da Costa Pinheiro ${ }^{V}$; Neiva Francenely Cunha Vieira ${ }^{V I}$
}

\begin{abstract}
RESUMO: O estudo objetivou identificar as concepções e ações de educação em saúde dos enfermeiros da estratégia de saúde da família (ESF), destacando aquelas relacionadas aos acidentes na infância e adolescência no domicílio. Estudo qualitativo com 10 enfermeiros da ESF, do Município de Imperatriz, Maranhão, Brasil. Os dados foram coletados por meio de entrevista semiestruturada, no mês de janeiro de 2012, e tratados sob a ótica da análise de conteúdo. Os resultados evidenciaram que a educação em saúde voltada para a prevenção de acidentes na infância e adolescência não faz parte da prática dos enfermeiros, sendo necessário abordar temas dessa natureza. Conclui-se que, em virtude dos acidentes serem recorrentes na área adstrita dos participantes, é necessário difundir a educação em saúde sobre o tema, a fim de reduzir os índices de acidentes e fortalecer o desejo dos usuários para a mudança de comportamentos em saúde.

Palavras-Chave: Enfermagem; educação em saúde; saúde da família; acidentes.
\end{abstract}

\begin{abstract}
This qualitative study aimed to identify nurses' conceptions of health education, and their related actions in the family health strategy, and to highlight those relating to accidents involving children and adolescents at home. Data were collected through semi-structured interviews of 10 family health strategy nurses in Imperatriz, Maranhão State, Brazil, in January 2012, and analyzed using content analysis. The results showed that nurses do not engage in health education directed to preventing accidents in childhood and adolescence, and that it is necessary to address issues of this nature. It was concluded that, as injuries are recurrent in the area assigned to the participants, health education on the subject is necessary in order to reduce accident rates and strengthen users' desire to change health-related behaviors.

Keywords: Nursing; health education; family health; accidents.

RESUMEN: El estudio tuvo como objetivo identificar los puntos de vista y las acciones de educación en salud de los enfermeros en la estrategia de salud de la familia (ESF), destacando los relacionados con accidentes en la infancia y la adolescencia en el hogar. Estudio cualitativo con 10 enfermeros de la ESF, de la ciudad de Imperatriz, Maranhão, Brasil. Los datos han sido recolectados a través de entrevista semiestructurada, en enero de 2012, y estudiados desde la perspectiva del análisis de contenido. Los resultados mostraron que la educación en salud volcada hacia la prevención de accidentes en la infancia y la adolescencia no forma parte de la práctica de los enfermeros, siendo necesario abordar cuestiones de esta naturaleza. Se concluye que, visto que los accidentes son frecuentes en el área relativa a los participantes, es necesario difundir la educación en salud sobre el tema, para reducir los índices de accidentes y fortalecer el deseo de los usuarios de cambiar los comportamientos de salud.
\end{abstract} Palabras Clave: Enfermería; educación en salud; salud de la familia; accidentes.

\section{INTRODUÇÃO}

Em todo o mundo, cerca de 800 mil crianças morrem em decorrência de acidentes. $\mathrm{O}$ trauma é a maior causa de mortalidade e incapacidade, sendo responsável por mais mortes do que todas as doenças combinadas ${ }^{1,2}$.

O Brasil aparece, juntamente com outros países, com elevado número de óbitos por acidentes em menores de 15 anos. Ao longo de um período de oito anos (2002 - 2009), estudos revelam que, no País, os acidentes na infância e adolescência foram responsáveis por 755.826 internações e 22.373 óbitos ${ }^{3}$.

No Estado do Maranhão, por exemplo, segundo informações da Secretaria Estadual de Saúde, nos anos de 2007 e 2009 foram registrados 678 atendimentos a crianças e adolescentes vítimas dos mais diversos tipos de acidentes, nos principais prontos-socorros do estado, representando 3,9\% do total

IEnfermeira. Mestre em Enfermagem. Docente da Universidade Federal do Maranhão/Centro de Ciências Sociais, Saúde e Tecnologia. Imperatriz, Maranhão, Brasil. E-mail: anacristina itz@hotmail.com.

"Enfermeira. Unidade Mista Dr. Neto Guterres. Alcântara, Maranhão, Brasil. E-mail: ester.cintia@hotmail.com.

IIIEnfermeira. Mestre em Cuidados Clínicos. Docente da Universidade Federal do Piauí. Picos, Piauí, Brasil. E-mail: analarissa2001@yahoo.com.br.

IVEnfermeira. Doutora em Enfermagem. Docente da Universidade Regional do Cariri. Crato, Ceará, Brasil. E-mail: alissankarine@gmail.com.

vEnfermeira. Doutora em Enfermagem. Docente da Universidade Federal do Ceará. Fortaleza, Ceará, Brasil. E-mail: neyva.pinheiro@yahoo.com.br.

VIEnfermeira. PhD in Health Education. Docente da Universidade Federal do Ceará. Fortaleza, Ceará, Brasil. E-mail: neivafrancenely@ @otmail.com. 
de 17.000 atendimentos realizados. Esta evidência sinaliza que o comportamento preventivo de acidentes com crianças e adolescentes deveria ser um dos compromissos dos profissionais de saúde, em virtude de sua incidência. Salienta-se que esses números podem ser subnotificados dado a inexistência de protocolos obrigatórios nos serviços de saúde do estado que identifiquem as causas dos acidentes e a faixa etária específica de ocorrência ${ }^{4,5}$.

A literatura é unânime ao apontar que os acidentes com crianças e adolescentes ocorrem, predominantemente, no ambiente doméstico, portanto, passíveis de serem evitados, especialmente os de maior recorrência, como quedas, queimaduras, intoxicações por medicamentos e/ou soluções químicas, maus tratos, mordidas de animais, ferimentos por armas branca e de fogo ${ }^{3,6}$.

A Convenção dos Direitos da Saúde da Criança e do Adolescente, o Estatuto da Criança e do Adolescente (ECA) e a Portaria MS/GM n ${ }^{\circ} 737$ - que aborda sobre a Política Nacional de Redução da Morbimortalidade por acidentes e violências - normalizam que deve ser assegurado a todos os setores da sociedade, em especial aos pais, às crianças e aos adolescentes, o conhecimento dos princípios básicos de saúde e de ações de prevenção de acidentes, recebendo apoio para a aplicação desses conhecimentos ${ }^{5,7}$.

Diante disto, é necessário incluir ações educativas junto às famílias para que conheçam as fases características de crescimento e desenvolvimento da criança e do adolescente, adotem comportamento saudável e promovam um ambiente doméstico favorável à saúde que contribua positivamente na redução de acidentes? Logo, cabe aos profissionais de saúde desempenhar seu papel como educador e dialogar com a população sobre conhecimentos das práticas preventivas, com vistas à promoção da saúde deste público ${ }^{8}$.

A principal filosofia da Estratégia Saúde da Família (ESF) é atuar na promoção da saúde da comunidade em geral, priorizando grupos vulneráveis. As repercussões dos acidentes com crianças e adolescentes, na família e na sociedade, devem ser consideradas como importante problema da área adstrita, passíveis de prevenção ${ }^{9-11}$. Além disto, danos permanentes ou mortes prematuras nesta etapa da vida podem levar à limitação e/ou perda total para atingir as expectativas individuais, sociais e espirituais que deem um sentido, alegria no desenvolvimento das capacidades para um viver saudável ${ }^{12}$.

Nesse sentido, o Ministério da Saúde recomenda medidas preventivas antes da manifestação de agravos, para minimizar a oportunidade de exposição aos fatores desencadeantes dos acidentes. Este estudo tem como objetivo identificar as concepções e ações de educação em saúde dos enfermeiros da ESF, relacionadas aos acidentes na infância e adolescência.

\section{ReVISÃo DE LITERATURA}

O trauma infantil, decorrente dos acidentes, tornou-se, atualmente, motivo de preocupação dos profissionais de saúde diante do crescente aumento. A cada 10 crianças vítimas de acidentes, uma necessita de atendimento pelo Sistema Único de Saúde (SUS), e outras 15 irão apresentar sequelas definitivas ${ }^{4}$.

No Brasil, os acidentes com crianças e adolescentes representam considerável impacto econômico com gastos hospitalares, com internação, dias de permanência - e em unidades de terapia intensiva -, o que significa $8 \%$ dos dispêndios com internações por todas as causas. Os principais acidentes envolvendo as crianças no Brasil são as quedas, sendo a causa preponderante de internação, quase $74 \%$ por causas externas em diferentes faixas etárias ${ }^{2,7}$.

A estimativa é de que em 2020, no Brasil, tenha-se um aumento para $22 \%$ de acidentes com crianças e adolescentes. Crianças menores de três anos de idade têm maior risco, devido à curiosidade, impulsividade e falta de maturidade para avaliar prováveis perigos, o que torna a necessidade de prevenção ainda mais urgente ${ }^{10,12}$.

É visível a necessidade de investimentos na prevenção de acidentes com crianças e adolescentes, tendo como passo inicial modificar a definição de que o acidente é uma injúria não intencional e dissociá-lo da imprevisibilidade e casualidade. As principais formas de atuação para a conscientização ocorrem por meio de ações educativas em saúde, que envolvem cuidados físicos, materiais e emocionais, além de alterações físicas do espaço domiciliar. Assim, os profissionais de saúde, sobretudo os integrantes da ESF, são desafiados a implementar ações educativas para prevenir os acidentes ${ }^{12}$.

\section{Metodologia}

Estudo qualitativo, desenvolvido no mês de janeiro de 2012, em unidades básicas de saúde (UBS), do Município de Imperatriz, Maranhão. Este possui 42 equipes da ESF, distribuídas em 30 UBS, em seis macrorregiões, sendo que seis unidades com mais de uma equipe participaram do estudo, representando a realidade de cada macrorregião. Ao todo, a área de cobertura abrange 2.700 famílias.

Os 42 enfermeiros das equipes da ESF do município receberam informações sobre o estudo e convite para participar. Apenas 10 enfermeiros aceitaram o convite de forma espontânea e assinaram o Termo de Consentimento Livre e Esclarecido.

A coleta dos dados foi realizada mediante entrevista semiestruturada, composta por 15 questões sobre características pessoais e profissionais dos informantes, concepções e ações de educação em saúde junto à 
família, capacitação para a prática de educação em saúde, concepções e ações educativas implementadas junto às famílias sobre acidentes na infância e adolescência. Uma UBS diferente das selecionadas para o presente estudo foi utilizada para testar o instrumento aplicado. As entrevistas foram previamente agendadas, no local de trabalho do participante, e desenvolvidas em salas disponibilizadas pela direção.

Para a análise dos dados, foi utilizado o referencial temático da análise de conteúdo ${ }^{13}$, contemplando a fase de pré-análise e exploração dos dados. Em seguida, os dados foram organizados em quatro categorias: educação em saúde: informação para prevenção; capacitação para prática de educação em saúde; concepções sobre acidente e suas causas; ocorrência de acidentes. As falas dos enfermeiros foram identificadas pela letra $\mathrm{E}$, seguida do número de ordem das entrevistas.

O projeto foi aprovado pelo Comitê de Ética em Pesquisa do Hospital Universitário da Universidade Federal do Maranhão, sob o parecer no 320/11, respeitando os preceitos da Resolução n ${ }^{\circ}$ 466/12.

\section{Resultados e Discussão}

\section{Caracterização dos participantes}

Os participantes do estudo estão na faixa etária entre 28 e 55 anos, oito são do sexo feminino, seis são casados e $6(60 \%)$ têm filhos. No que se refere ao tempo de formação, possuem, em média, 10 anos, ressaltando-se que oito relataram possuir uma das seguintes especialidades: saúde da família, saúde coletiva e enfermagem obstétrica. $\bigcirc$ tempo de atuação profissional na ESF variou entre 8 meses e 12 anos. Observe-se que a maioria dos participantes possui formação em subárea de conhecimento de enfermagem para atuar na ESF. As categorias emergentes dos discursos dos enfermeiros são analisadas a seguir.

\section{Educação em saúde: informação para prevenção}

As falas dos participantes indicam uma concepção de educação em saúde e de processo educativo sem a criação de oportunidades para o diálogo e a troca de saberes entre os sujeitos, com enfoque prioritário na prevenção de doenças mediante palestras, conforme pode ser vislumbrado nos relatos:

Educação em saúde são orientações nas palestras para promover saúde da população, evitando as doenças. (E2)

É prevenir a população das doenças através de palestras. (E4)

Educação em saúde é base da saúde da família [...] porque através dela podemos orientar as famílias sobre como prevenir as doenças. (E7)

É informar a comunidade sobre a melhor forma de se cuidar, prevenir doenças e promover saúde. (E8)
A educação em saúde propõe o desenvolvimento do indivíduo ou da comunidade como um agente de mudanças no processo saúde-doença, por meio de diálogos com reflexão, com sensibilidade para o senso crítico, aspectos não percebidos nos relatos dos participantes. Houve predominância de concepções superadas de educação em saúde, como transmissão de informações, com base na pedagogia tradicional ${ }^{13}$. Consoante aos resultados, estudos também apontam a ausência de práticas educativas problematizadoras, com enfoque na promoção da saúde e participação dos sujeitos na $\mathrm{ESF}^{7,8}$.

Educar em saúde não deve ser um processo que almeje exclusivamente a prevenção de doenças, mas usada como estratégia para troca de saberes e experiências entre profissionais de saúde e usuários, a fim de fortalecer as potencialidades individuais ou coletivas, fator tido como primordial à mudança de comportamento. Logo, é preciso ter cuidado para que a educação em saúde não se transforme em transmissão de conhecimentos apenas pelo educador ${ }^{14,15}$.

Outro aspecto importante: cabe ao educador em saúde promover estratégias para colaborar com os usuários na compreensão dos processos de saúde e adoecimento e, dependendo da necessidade, deverá intervir individualmente e/ou coletivamente, de forma autônoma, criativa, e valorizando o cotidiano dos clientes. A utilização do diálogo é ferramenta importante para o processo educativo, embora seja, ainda, um grande desafio para ambas as partes, profissionais e sujeitos ${ }^{14}$.

Apesar de a maioria possuir especialização na área de saúde coletiva, as suas práticas educativas revelam a necessidade de mais aporte teórico e metodológico para que o exercício profissional estimule mais participação e discussões sobre educação em saúde com os usuários dos serviços.

\section{Capacitação para prática de educação em saúde}

A equipe reconhece a falta de formação específica para implementar, com qualidade, a educação em saúde aos usuários. Logo, deve estar qualificada para atuar nesse contexto, planejando, executando e avaliando as ações educativas, conforme a realidade das comunidades, a partir das demandas dos usuários. Os depoimentos que seguem demonstram essa lacuna:

Desde que estou aqui, nesta unidade, teve um treinamento sobre educação em saúde em 2008. (E2)

De um modo geral, faz tempo que aqui não recebemos nenhuma qualificação voltada para educação em saúde. (E3)

No município temos acompanhamento de educação continuada para atualizações sobre vacinas, hepatites virais, hanseníase [...]. (E8)

Na atenção primária de saúde, as ações de educação em saúde são inerentes ao processo de trabalho da ESF. Entretanto, é preciso que seja incluída, na gestão 
do trabalho em saúde dos profissionais, oportunidades de formação que contemplem conhecimentos, estratégias, tecnologias em um processo amplo e contínuo, considerando as vivências, crenças, experiências, valores, sentimentos e percepções da comunidade. É cada vez mais necessário que a gestão dos serviços de saúde insira, nos processos de trabalho em atenção primária, planejamento, implementação e avaliação das ações educativas, a partir de constante capacitação teórico- prática ${ }^{7}$. A ausência de capacitação contínua dos profissionais de saúde limita a amplitude das ações pertinentes à educação em saúde, principalmente quando assumem apenas as tradicionais ações normatizadas ${ }^{8,16}$.

Os participantes informaram, ainda, que as ações de educação em saúde desenvolvidas se limitam a focar datas comemorativas específicas na área da saúde e ao acompanhamento de grupos formados na UBS, o que reforça a ideia de que os profissionais vinculam educação em saúde às ações normativas e prescritivas de estilo de vida, com enfoque nos programas de saúde, conforme se observa a seguir.

Tento priorizar acontecimentos referentes ao mês: dia da criança, dia da mulher, amamentação. (E3)

O assunto mais falado é hipertensão e diabetes, [...] sobre alimentação, exercício físico; área da criança, ninguém fala nada. (E5)

Ação eu faço com o hiperdia, [...] palestras nas escolas marco esporadicamente, dependendo do que vou trabalhar naquele mês. (E8)

As falas dos profissionais da ESF estão carregadas de determinações intervencionistas e com olhar reducionista do processo saúde-doença.

Ainda que reconheçam a importância sobre a temática acidentes, os participantes priorizam temas em função da agenda demandada pelos programas do Ministério da Saúde como diabetes e hipertensão. Sem desconsiderar a relevância destas atividades em função da vigilância em saúde, nada impede que os profissionais se mobilizem para atender a demandas educativas específicas da área adstrita.

As ações de educação em saúde precisam ser vistas como estratégias direcionadas à prevenção, promoção, cura e reabilitação. Nesse sentido, as práticas educativas não podem ter apenas enfoque preventivo, precisam ser mais abrangentes e integralizadoras, estimulando o desenvolvimento pessoal dos sujeitos envolvidos. A proposta de práticas educativas a partir das necessidades dos usuários insere-se no discurso emergente de educação em saúde - o modelo dialógico, que em oposição ao modelo tradicional, trabalha sob a perspectiva de sujeitos autônomos e participantes das práticas de saúde. Logo, rever as práticas de educação em saúde, diante dos novos padrões, torna-se imprescindível para adotar novas visões, bem como repensar a metodologia de trabalho atual ${ }^{15,17}$.

\section{Concepções sobre acidente e suas causas}

O conhecimento sobre acidentes é importante para a tomada de decisão, a fim de preveni-los e promover a saúde da criança e do adolescente. Sobre este aspecto, os participantes assim se revelaram:

Acidentes são situações que colocam em risco a integridade do ser humano, geralmente poderiam ser evitados [...] se houvesse mais prevenção por parte dos familiares, o número de casos iria diminuir. (E3)

Acidente é tudo que possa causar lesão da integridade física e mental das crianças e dos adolescentes. (E5)

Eu acho que acidente é tudo aquilo que não se espera que aconteça, que possa causar danos à saúde [...] A mãe descuidada também é um grande fator para causar acidentes, além da falta de informação [...] (E6)

Acidentes são eventos previsíveis, resultando em danos e até morte. Os acidentes são concebidos como passíveis de serem evitados por meio de medidas que envolvam cuidados materiais, emocionais, físicos e sociais, destacando-se, dessa forma, a necessidade de prevenção ${ }^{3,4}$. No entanto, qualquer mudança de comportamento ocorre com a decisão do usuário, considerando seu contexto econômico, social e cultural. Consoante aos resultados, alguns estudos também apontam a ausência de práticas educativas problematizadoras, com enfoque na promoção da saúde e participação dos usuários na ESF ${ }^{7-10}$.

Nessa perspectiva, quando ocorre um acontecimento negativo no âmbito familiar, os profissionais buscam descobrir quem é o responsável, o culpado. Frequentemente, as atribuições de responsabilidade relacionam-se simultaneamente à causalidade e às obrigações das pessoas. Comportamentos negligentes das famílias com suas crianças e adolescentes contribuem para a aproximação precoce aos ambientes de risco, tornando-os vulneráveis às diversas formas de acidentes. Além disso, podem ser respostas diretas das dificuldades que vivenciam no cuidar dos filhos, reflexos, geralmente, das condições sociais a que são subjugadas ${ }^{15}$.

Ao atribuírem de maneira recorrente aos pais a responsabilidade das causas do acidente, sem aprofundar o estudo das questões sociais das famílias, tais como sobrecarga profissional e do papel materno, ausência de rede de apoio social, falta de instrução, abuso de substâncias psicoativas, os profissionais responsabilizam as famílias, sem que haja sensibilização, ações e prontidão para intervir de forma adequada nas realidades vividas.

Os acidentes com crianças e adolescentes são potencializados pela inobservância, ausência de comportamento preventivo das famílias e falha na vigilância do adulto responsável. Na maioria das ocasiões, os responsáveis pelos menores desconhecem as fases de crescimento, desenvolvimento e suas necessidades, o que favorece a ocorrência de acidentes. Nesse con- 
texto, justifica-se a implementação de ação educativa voltada para a prevenção de acidentes, envolvendo todos os integrantes da família que convivem e dividem as atribuições dos cuidados ${ }^{10}$.

\section{A ocorrência de acidentes}

Os enfermeiros reconhecem a ocorrência de acidentes na área adstrita, relatando serem frequentes entre crianças e adolescentes, conforme se pode depreender das falas:

Aqui, uma criança chegou a falecer, porque tomou vários comprimidos de sulfato ferroso [...]. (E3)

Temos muitos casos de queimaduras, atropelamentos, quedas de motos. (E5)

Chegam aqui, principalmente, por causa de quedas: rede, cama, escadas, bicicleta [...], mas também por queimaduras. (E6)

Entre as causas predominantes dos acidentes relatadas, a literatura destaca como mais representativas as quedas, com média de $52,4 \%$ das internações em todo o País 5 . Segundo levantamento bibliográfico realizado, não há publicações sobre essa temática referente à Região Sudoeste Maranhense, que corresponde ao Município de Imperatriz, não havendo, inclusive, um instrumento formal para registrar a notificação destes agravos. Isto revela a necessidade do registro desses dados, devido à complexidade dos acidentes com crianças e adolescentes, sendo relevante que os responsáveis pela elaboração e implementação de políticas aplicadas à saúde e prevenção de acidentes tomem decisões para evitar causas, sequelas, sofrimentos e, até mesmo, morte.

Há condições no lar que favorecem a ocorrência dos acidentes, como os fatores químicos, físicos, biológicos, estruturais, culturais. Trabalhar educação em saúde na atenção primária significa promover conhecimentos sobre segurança e sensibilização para adoção de comportamentos mais seguros no ambiente doméstico e, assim, contribuir para a redução da incidência dos acidentes ${ }^{2,6}$.

Destaca-se que os fatores determinantes dos acidentes com crianças e adolescentes reportam-se às particularidades das comunidades. Assim, é necessário que qualquer reflexão ou proposta de ação se baseie na realidade local, quer seja do ponto de vista epidemiológico, social ou cultural, levando em conta os riscos inerentes ao ambiente e ao estágio de desenvolvimento neuropsicomotor de ambos, criança e adolescente ${ }^{8,17}$.

Embora seja conhecida a ocorrência de acidentes envolvendo crianças e adolescentes, as ações de educação em saúde da UBS não respondem à demanda para a prevenção. É surpreendente a negação dos participantes sobre a realização de atividades específicas para este fim. As falas expressam esta realidade:
Educação especificamente sobre acidente com criança não, muito menos com adolescente. Eu nunca fiz nada na unidade de saúde sobre esse assunto. (E2)

Esse é um tema interessante, chega muita criança com objetos no nariz, que caiu, com queimadura, mas nunca fiz palestra sobre isso não. (E3)

Nunca fiz palestra direcionada a acidentes com criança e adolescente. [...] aconteceu aqui um caso muito grave, que passou na TV, por afogamento em uma bacia. (E4)

Eu nunca falei sobre isso. A educação em saúde não tem que ser responsabilidade só do enfermeiro [...]. (E6)

processo de elaboração de ações educativas para prevenção dos acidentes precisa ser compreendido e praticado de forma continuada, especialmente quando a incidência é frequente. Dessa forma, as ações voltadas para tal problemática devem estar em consonância com o conceito de prevenção, que é caracterizado pela ação antecipada, cujo objetivo é interceptar ou anular a evolução dos acontecimentos, evitando que algum dano aconteça, mediante o exercício de cuidados físicos, materiais, emocionais e sociais ${ }^{12}$.

Sem desconsiderar a importância do atendimento das demandas programáticas da ESF, atuar com o objetivo de promover saúde no âmbito familiar implica conhecimento do cotidiano dos seus membros e atribuição específica dos integrantes da equipe. Estudos envolvendo famílias, realizados em diversos cenários de atuação dos profissionais de saúde, como, por exemplo, no hospital, mostrou aumento significativo do aprendizado de familiares sobre o tema acidentes, demonstrando potencial satisfatório da ação educativa no contexto hospitalar. Logo, torna-se proposta recomendada a outros cenários, como as unidades da ESF. O papel do enfermeiro implica uma visão integral da criança, do adolescente e de sua família, relacionada aos fatores individuais, sociais e programáticos que interferem na ocorrência e possibilidade de prevenção dos acidentes ${ }^{10,14}$.

Estudos que enfatizam a magnitude dos acidentes na infância e adolescência sugerem a necessidade de ações de educação em saúde junto a esse público, família, comunidade e sociedade, no sentido de alertar para riscos e necessidade de adotar comportamentos seguros em relação aos ambientes passíveis de acidentes. Portanto, tem-se na promoção da saúde a maneira mais eficaz para reduzir elevados indicadores de acidentes na infância e adolescência ${ }^{15}$.

A Carta de Otawa traz o conceito de promoção da saúde, que significa fornecer às populações condições para torná-las capazes de melhorar a saúde e exercer controle sobre ela. Nesse contexto, formaliza a criação de ambientes favoráveis à saúde como um dos cincos campos da promoção da saúde. Tal ambiente favorável à saúde não se relaciona apenas à proteção do meio ambiente e à conservação dos recursos na- 
turais, mas à ambiência onde ocorrem as relações de trabalho entre enfermeiros, crianças, adolescentes e suas famílias, possibilitando observar os determinantes biológicos, sociais e culturais, buscando a integralidade do cuidado no domicílio ${ }^{16-18}$.

\section{Conclusão}

Conclui-se que as ações educativas devem ser realizadas junto a todos os envolvidos na prevenção de acidentes - crianças, adolescentes, família e sociedade -, no sentido de alertar sobre riscos e adoção de comportamentos seguros em relação aos ambientes e às fases de desenvolvimento infantil e do adolescer.

O estudo leva a compreender a necessidade de os profissionais de saúde, como facilitadores da aquisição de conhecimento em saúde, desenvolverem ações educativas voltadas para a prevenção dos acidentes com crianças e adolescentes, preferencialmente, englobando todos os cenários possíveis, a partir do domicílio, inclusive os determinantes socioeconômicos.

$\mathrm{O}$ estudo apresenta como limitações a reduzida adesão à pesquisa dos enfermeiros da ESF do município e a inexistência de ações educativas dos profissionais pesquisados sobre prevenção de acidentes, ainda que reconheçam a ocorrência frequente desses eventos. Recomenda-se o desenvolvimento, na comunidade, de programas de educação e prevenção relacionados aos riscos e danos dos acidentes, para auxiliar no desenvolvimento de potencialidades e empoderamento dos sujeitos.

\section{REFERÊNCIAS}

1.Mascarenhas MDM, Malta DC, Silva MMA, Lima CM, Carvalho MCO, Oliveira VLA. Violência contra a criança: revelando o perfil dos atendimentos em serviços de emergência, Brasil, 2006 e 2007. Cad Saúde Pública. 2010; 26:347-57.

2.Malta DC, Mascarenhas MDM, Silva MMA, Macário EM. Perfil dos atendimentos de emergência por acidentes envolvendo crianças menores de dez anos - Brasil, 2006 a 2007. Ciênc saúde coletiva. 2009; 14:1669-79.

3.Ministério da Saúde (Br). Secretaria de Políticas de Saúde. Política Nacional de Redução da Morbimortalidade por acidentes e violência. Rev Saúde Pública. 2010; 34:427-30. 4.Andrade LM, Caetano JA, Vieira LJES, Lima M. Caracterização de crianças vítimas de acidentes e violências admitidas em unidade de terapia intensiva pediátrica. Rev Enferm UFPE. 2010; 4:1401-9.

5.Cervera DPP, Parreira BDM, Goulart BF. Educação em saúde: percepção dos enfermeiros da atenção básica em Uberaba (MG). Ciênc saúde coletiva. 2011; 16:1547-54. 6. Malta DC, Mascarenhas MDM, Bernal RTI, Viegas APB, Sá NNB, Junior JBS. Accidents and violence in childhood: survey evidence of emergency care for external causes - Brazil. Ciênc saúde coletiva. 2012; 17:2247-58.

7.Gimeniz-Paschoal SR, Pereira DM, Nascimento EN. Efect of an educative action on relatives' knowledge about childhood burns at home. Rev Latino-Am Enfermagem. 2009; 17:341-6.

8.Silva MAI, Pan R, Meo L, Bortoli OS, Nascimento LC. Perfil dos atendimentos a crianças e adolescentes vítimas de causas externas de morbimortalidade, 2000-2006. Rev Gaúcha Enferm. 2010; 31:351-8.

9.Smith JB, Cho Tang K, Don Nutbeam. Who health Promotion glossary: new terms. Health Promotion International. 2006; 21:340-5.

10.Fernandes MCP, Backes VMS. Educação em saúde: perspectivas de uma equipe da estratégia saúde da família sob a óptica de Paulo Freire. Rev Bras Enferm. 2010; 63:567-73.

11.Acioli S, David HMSL, Farid MGA. Educação em saúde e a enfermagem em saúde coletiva: reflexões sobre a prática. Rev enferm UERJ. 2012; 20:533-6.

12.Machado MFAS, Vieira NFC. Health education: the family health teams' perspective and clients' participation. Rev Latino-Am Enfermagem. 2009; 17:174-9. 13. Bardin L. A análise de conteúdo. $3^{\mathrm{a}}$ ed. Lisboa (Pt): Edições 70; 2011.

14.Fernandes MCP, Backes VMS. Educação em saúde: perspectivas de uma equipe da estratégia saúde da família sob a óptica de Paulo Freire. Rev Bras Enferm. 2010; 63:567-73.

15.Roecker S, Marcon SS. Educação em saúde na estratégia saúde da família: o significado e a práxis dos enfermeiros. Esc Anna Nery. 2011; 15:701-9.

16.Ximenes Neto FRG, Aguiar DT, Martins FR, Silva RCC, Cunha ICK. Práticas do enfermeiro da estratégia saúde da família na atenção à saúde da criança, Cariri- Ceará. Rev Soc Bras de Enferm Ped. 2011; 11:9-16. 17.Vieira LJES, Carneiro RCMM, Frota MA, Gomes ALA, Ximenes LB. Ações e possibilidades de prevenção de acidentes com crianças em creches de Fortaleza, Ceará. Ciên saúde coletiva. 2009; 14:1687-97.

18. Buss PM. Promoção da saúde e saúde pública. Rio de Janeiro: Escola Nacional de Saúde Pública; 1996. 